\title{
Towards multiscale modeling of magnetic materials: Simulations of $\mathrm{FePt}$
}

\author{
N. Kazantseva, ${ }_{1}^{1}$ D. Hinzke, ${ }^{1}$ U. Nowak, ${ }^{1, *}$ R. W. Chantrell, ${ }^{1}$ U. Atxitia, ${ }^{2}$ and O. Chubykalo-Fesenko ${ }^{2}$ \\ 'Department of Physics, University of York, York YO10 5DD, United Kingdom \\ ${ }^{2}$ Instituto de Ciencia de Materiales de Madrid, CSIC, Cantoblanco, 28049 Madrid, Spain
}

\begin{abstract}
The established methods for the numerical evaluation of magnetic material properties exist only in certain limits, including first-principles methods, spin models, and micromagnetics. In the present paper, we introduce a multiscale modeling approach, bridging the gaps between the three approaches above. The goal is to describe thermodynamic equilibrium and nonequilibrium properties of magnetic materials on length scales up to micrometers, starting from first principles. In the first step, we model, as an example, bulk FePt in the ordered $\mathrm{Ll}_{0}$ phase by using an effective, classical spin Hamiltonian that was constructed earlier on the basis of firstprinciples methods. The next step is to simulate this spin model by using the stochastic Landau-LifshitzGilbert equation. The temperature dependent micromagnetic parameters, which are evaluated with these atomistic simulations, are consequently used to develop a many macrospin micromagnetic approach, based on the Landau-Lifshitz-Bloch equation. As an example, we calculate the magnetization dynamics following a picosecond heat pulse resembling pump-probe experiments.
\end{abstract}

PACS number(s): $75.40 . \mathrm{Gb}, 75.40 . \mathrm{Mg}, 75.75 .+\mathrm{a}$

\section{INTRODUCTION}

An increasing amount of research is currently focusing on the thermodynamic equilibrium and nonequilibrium behavior of ferromagnetic materials. The motivations are manifold, reaching from the problem of thermal stability of information magnetically stored on length scales of only nanometers to the understanding of laser-induced spin dynamics in the picosecond regime, ${ }^{1-11}$ opening new perspectives for applications in magnetic storage devices and spintronics.

At the moment, well-established methods for the investigation of magnetic material properties exist only in certain limits, including mainly so-called first-principles methods, spin models, and micromagnetics. The basis of most of the numerical calculation of magnetization dynamics is a micromagnetic approach (for a review, see Ref. 12) that considers the magnetization of either a small particle or a discrete magnetic nanoelement as a vector of a fixed length (a macrospin) following the Landau-Lifshitz-Gilbert (LLG) equation of motion. Even though the LLG equation is (partly) phenomenological, this approach turns out to be very successful as far as descriptions of its ground state domain structures and its zero temperature dynamics are concerned. The method is capable of describing the properties of magnetic systems of sizes up to the micrometer regime. For an extension to finite temperatures, the LLG equation is usually augmented by a stochastic term. ${ }^{13}$ However, contrary to the situation with atomic spins, there is no reason to assume a fixed magnetization length for nanoelements at nonzero temperature, so that this approach lacks the essential physics to describe relevant thermodynamic phenomena.

When compared to this micromagnetic approach, the use of atomistic spin models for the description of magnetic materials has advantages as well as disadvantages. ${ }^{14}$ The main disadvantage is that due to the atomic resolution, the system size is clearly restricted to a nanometer scale. The advantages are (i) realistic lattice structures can be taken into account without assuming a continuous magnetization, (ii) the mod- eling of para-, ferri-, ferro-, or antiferromagnets, and even heterostructures composed of several of these different materials including interface properties is straightforward, and most importantly, (iii) finite temperatures can be taken into account without cutting the spin wave spectra due to the discretization.

However, both of the methods described above usually rest on models wherein material parameters, such as exchange integrals and anisotropy constants, are more or less treated as fitting parameters. Methods that avoid this and directly calculate material properties are called "firstprinciples methods." These methods usually base on spin density functional theory (SDFT) and directly calculate magnetic ground state properties for a given material from its electronic structure. However, these methods are restricted to either translationally invariant (infinite) systems or small groups of atoms. The purpose of this paper is to introduce a hierarchical multiscale modeling method for magnetic materials, bridging the gaps between the three approaches above, with the goal to describe thermodynamic equilibrium and nonequilibrium properties of magnetic materials, starting from first principles on length scales from the single atom reaching to micrometers.

In bridging the gap between micromagnetics and spin models, we note the occurrence of several important effects of the macrospin dynamics at elevated temperatures: ${ }^{15}$ first, that during switching, the magnetization magnitude is not constant in time; second, that the longitudinal relaxation time sharply increases approaching the Curie temperature; and third, that at the same time the transverse relaxation time sharply decreases. Within a (micromagnetic) macrospin approach, these phenomena cannot be described in terms of the conventional LLG equation of motion. However, they are in agreement with the Landau-Lifshitz-Bloch (LLB) equation, ${ }^{15}$ which was recently derived by Garanin within the mean-field approximation from the classical FokkerPlanck equation for individual spins interacting with a heat bath. ${ }^{16}$ Therefore, we use the LLB equation as a new basis for micromagnetics at elevated temperatures and demon- 
strate the capabilities of a micromagnetic LLB equation, by investigating the temperature dependent magnetization dynamics and comparing to results from spin model simulations.

Regarding the gap between spin models and firstprinciples methods, we note that a systematic construction and parameterization of a spin model Hamiltonian for a given material on the basis of first-principles calculations is a challenge. Major problems include a non-Heisenberg form of the exchange and a delocalized character of the magnetic moment. Nevertheless, some pioneering calculations of exchange integrals and even anisotropy constants (besides the more common calculations of the atomic magnetic moment) with the aim to derive an effective classical spin Hamiltonian exist in the literature. In the following, we will use a spin model for FePt that was derived earlier ${ }^{17}$ and the capabilities of which have been demonstrated elsewhere. ${ }^{17-19}$

The structure of our paper is as follows: in Sec. II, we introduce the LLB equation and point out where the information from spin model simulation comes in. In Sec. III, we introduce the spin model itself that contains the information from SDFT calculation and calculate the quantities needed for the simulation of the LLB equation. After that, our results are presented in Sec. IV, including a simulation of pumpprobe experiments with a macrospin model capable of predictions both on the picosecond time scale of fast demagnetization and the nanosecond time scale of precession and magnetization recovery. Finally, we summarize and give an outlook.

\section{LANDAU-LIFSHITZ-BLOCH EQUATION}

An equation of motion for macrospins allowing for longitudinal relaxation was derived by Garanin ${ }^{16}$ within a meanfield approximation from the classical Fokker-Planck equation for atomistic spins interacting with a heat bath. The resulting "Landau-Lifshitz-Bloch equation" has been shown to be able of describing linear domain walls, a domain wall type with a nonconstant magnetization length. These results are consistent with measurements of the domain wall mobility in YIG crystals close to $T_{C}$ (Ref. 20) and with recent atomistic simulations. ${ }^{21}$ Furthermore, the predictions for the longitudinal and transverse relaxation times were successfully compared to atomistic simulations. ${ }^{15}$ Consequently, we use this equation in the following for the thermodynamics simulation of macrospins.

The LLB equation can be written in the form

$$
\begin{aligned}
& \dot{\mathbf{m}}_{i}=-\tilde{\gamma}\left(\mathbf{m}_{i} \times \mathbf{H}_{\mathrm{eff}}^{i}\right)+\frac{\tilde{\gamma} \alpha_{i}}{m_{i}^{2}}\left[\mathbf{m}_{i} \cdot\left(\mathbf{H}_{\mathrm{eff}}^{i}+\zeta_{i}\right)\right] \mathbf{m}_{i} \\
& -\frac{\tilde{\gamma} \alpha_{\perp}}{m_{i}^{2}}\left\{\mathbf{m}_{i} \times\left[\mathbf{m}_{i} \times\left(\mathbf{H}_{\mathrm{eff}}^{i}+\zeta_{\perp}\right)\right]\right\} .
\end{aligned}
$$

Besides the usual precession and relaxation terms, the LLB equation contains another term that controls longitudinal relaxation. Hence, $\mathbf{m}_{i}$ is a spin polarization that is not assumed to be of constant length and even its equilibrium value $m_{e}(T)$ is temperature dependent. $\alpha_{\|}$and $\alpha_{\perp}$ are dimensionless longitudinal and transverse damping parameters.
Note that when compared to Eq. (12), it is $\tilde{\gamma}=\gamma /\left(1+\lambda^{2}\right)$, where $\gamma$ is the gyromagnetic ratio.

Thermal fluctuations ${ }^{22}$ are included as an additional noise term $\zeta_{l}^{i}(t)$ with $l=\perp, \|,\left\langle\zeta_{l}^{i}(t)\right\rangle=0$, and

$$
\left\langle\zeta_{i}^{i, \nu}(0) \zeta_{i}^{j, \eta}(t)\right\rangle=\frac{2 k_{B} T}{\tilde{\gamma} \alpha_{l} M_{\mathrm{s}}^{0} \Delta^{3}} \delta_{\nu \eta} \delta_{i j} \delta(t),
$$

where $i, j$ denotes lattice sites and $\nu, \eta$ denotes the Cartesian components. Here, $\Delta^{3}$ is the volume of the micromagnetic cell and $M_{s}^{0}$ is the value of the spontaneous magnetization at zero temperature.

The LLB equation is valid for finite temperatures and even above $T_{C}$, although the damping parameters and effective fields are different below and above $T_{C}$. For $T \leq T_{C}$, the damping parameters are

$$
\alpha_{\mathrm{l}}=\lambda \frac{2 T}{3 T_{C}} \quad \alpha_{\perp}=\lambda\left(1-\frac{T}{3 T_{C}}\right),
$$

and for $T \geq T_{C}$ the damping parameters are equal,

$$
\alpha_{\perp}=\alpha_{1 !}=\frac{2 \lambda T}{3 T_{C}} .
$$

The effective fields $\mathbf{H}_{\mathrm{eff}}^{i}=-\frac{1}{M_{s}^{0}} \frac{\delta f}{\delta \mathbf{m}_{i}}$ with free energy density $f$ are given by ${ }^{16}$

$$
\mathbf{H}_{\mathrm{eff}}^{i}=\mathbf{H}+\mathbf{H}_{A}^{i}+\mathbf{H}_{\mathrm{ex}}^{i}+ \begin{cases}\frac{1}{2 \tilde{\chi}_{i}}\left(1-\frac{m_{i}^{2}}{m_{e}^{2}}\right) \mathbf{m}_{i} & T \leq T_{C} \\ -\frac{1}{\tilde{\chi}_{i}}\left(1+\frac{3 T_{C} m_{i}^{2}}{5\left(T-T_{C}\right)}\right) \mathbf{m}_{i} & T \geq T_{C}\end{cases}
$$

where the anisotropy field is

$$
\mathbf{H}_{A}^{i}=-\frac{\left(m_{x}^{i} \mathbf{e}_{x}+m_{y}^{i} \mathbf{e}_{y}\right)}{\tilde{\chi}_{\perp}},
$$

which makes the $z$ axis the easy axis of the model and the exchange field

$$
\mathbf{H}_{\mathrm{ex}}^{i}=-\frac{A}{m_{e}^{2}} \frac{2}{M_{s}^{0} \Delta^{2}} \sum_{j \in \operatorname{neigh}(i)}\left(\mathbf{m}_{j}-\mathbf{m}_{i}\right) .
$$

Note that within the context of the LLB equation, field components parallel to the local magnetic moment are relevant since they can change the length of the magnetization vector. However, in order to conserve the mean length of the magnetization, the effective fields must vanish at equilibrium. The susceptibilities $\widetilde{\chi}_{l}$ are defined by $\widetilde{\chi}_{l}=\partial m_{l} / \partial H_{l}$. Note that at lower temperatures, the perpendicular susceptibility $\tilde{X}_{\perp}$ is related to the anisotropy anisotropy $K$ via $\widetilde{X}_{\perp}=M_{s}^{0} m_{e}^{2} /(2 K){ }^{16}$

In these equations, $\lambda$ is a microscopic parameter that characterizes the coupling of the individual, atomistic spins with the heat bath. Note that, even when assuming $\lambda$ to be temperature independent, the macroscopic damping parameters of the LLB equation turn out to be temperature dependent. ${ }^{15}$ In the limit $T \rightarrow 0$, the longitudinal damping parameter $\alpha_{\|}$ 
vanishes and with $|\mathbf{m}|=m_{e}(0)$ (the zero temperature equilibrium magnetization) the LLB equation goes over to the usual LLG equation.

A problem for the application of this equation is that one has to know the spontaneous equilibrium magnetization $m_{e}(T)$, the perpendicular $\left[\tilde{\chi}_{.}(T)\right]$ and parallel $\left[\tilde{\chi}_{(}(T)\right]$ susceptibilities as well as the exchange stiffness $A(T)$. In former publications, ${ }^{22,23}$ these were taken from a mean-field approximation. However, here we want to go beyond this approach and separately calculate these functions from a Langevin dynamics simulation of an atomistic spin model. As an example, we choose a model for FePt that was introduced earlier and that is meanwhile well established in literature. ${ }^{17-19,24}$ Since this model was derived from first principles, a direct link is made from SDFT calculations via a spin model to our macrospin simulations.

\section{ATOMISTIC SPIN MODEL FOR FePt}

FePt is intensively investigated due to its potential application as ultrahigh density recording media. ${ }^{25}$ In former publications, we modeled bulk FePt in the layered $L 1_{0}$ phase. The model was constructed on the basis of first-principles calculations of noncollinear configurations calculated by using constrained the local spin density functional theory ${ }^{26}$ and site-resolved magnetocrystalline anisotropy. ${ }^{27}$ The fundamental interactions at the electronic level are strongly modified by the $L 1_{0}$ structure. In particular, it has been shown ${ }^{17}$ that the Fe moments can be considered as localized, while the $\mathrm{Pt}$ induced moments have to be treated as essentially delocalized. Nevertheless, it is possible to construct a classical spin Hamiltonian involving only the Fe degrees of freedom, with the introduction of a two-ion anisotropy term and a modified exchange term. ${ }^{17}$ In order to verify the form of the Hamiltonian, especially the two-ion anisotropy, equilibrium data for the temperature dependence of the anisotropy constant were compared to the experimental data and it was shown ${ }^{17}$ that this model successfully describes the critical temperature and the anomalous temperature dependence of the uniaxial anisotropy energy constant $K_{1}$ experimentally found in this ordered alloy. ${ }^{28,29}$ Further investigations of the model include its dynamic behavior, ${ }^{18}$ the orientation dependence of domain wall properties, ${ }^{19}$ a direct consequence of the fact that interaction within the Fe planes are larger than those perpendicular to the planes, and the temperature dependence of the domain wall profiles. ${ }^{19,24}$

In the following, we consider the full Hamiltonian, which is described in detail in Ref. 17, including Zeeman energy and dipole-dipole coupling,

$$
\begin{aligned}
\mathcal{H}= & -\sum_{i<j}\left(\tilde{J}_{i j} \mathbf{S}_{i} \cdot \mathbf{S}_{j}+d_{i j}^{(2)} S_{i j}^{z} S_{j}^{z}\right)-\sum_{i} d^{(0)}\left(S_{i}^{z}\right)^{2} \\
& -\sum_{i<j} \frac{\mu_{0} \mu_{s}^{2}}{4 \pi} \frac{3\left(\mathbf{S}_{i} \cdot \mathbf{e}_{i j}\right)\left(\mathbf{e}_{i j} \mathbf{S}_{j}\right)-\mathbf{S}_{f} \mathbf{s}_{i}}{r_{i j}^{3}}-\sum_{i} \mu_{s} \mathbf{B} \cdot \mathbf{S}_{i},
\end{aligned}
$$

where $\mu_{s}$ is the atomistic magnetic moment. This spin Hamiltonian is expressed only in terms of $\mathrm{Fe}$ degrees of freedom. The first sum represents the exchange energy of the Fe moments with effective exchange interactions constant $\tilde{J}_{i j}$ given by

$$
\tilde{J}_{i j}=J_{i j}+I\left(\frac{\chi_{\nu}}{M_{v}^{0}}\right)^{2} \sum_{v} J_{i v} J_{j v^{*}}
$$

Here, $J_{i j}$ represent the exchange parameters between the $\mathrm{Fe}$ moments and the $J_{i v}$ the ones between $\mathrm{Fe}$ and $\mathrm{Pt}$ sites (the $\mathrm{Pt}-\mathrm{Pt}$ interaction is neglected). The intra-atomic interactions are described by the parameter $\tilde{I} \cdot \chi_{v}$ that represents the local Pt susceptibility, while the Pt magnetic moment in the ferromagnetic state are given by $M_{v}^{0}$. The sum over $\nu$ is over sites of the Pt sublattice only. Interestingly, the interactions $\bar{J}_{i j}$ depend on the orientation within the crystal: Fe-Fe interactions within the $\mathrm{Fe}$ planes are stronger than those perpendicular to the planes via the Pt moments.

The exchange interactions $\widetilde{J}_{i j}$ (and consequently also the $\left.d_{i j}^{(2)}\right)$ are taken into account up to a distance of 5 unit cells until they are finally small enough to be neglected. The twoion anisotropy parameters

$$
d_{i j}^{(2)}=k_{\mathrm{Pt}}^{(0)}\left(\frac{\chi_{\nu}}{M_{v}^{0}}\right)^{2} \sum_{v} J_{i v} J_{j \nu},
$$

are the dominant contributions to the uniaxial anisotropy energy in relation to the single-ion term

$$
d_{i}^{(0)}=k_{\mathrm{Fe}}^{(0)}+k_{\mathrm{Pt}}^{(0)}\left(\frac{\chi_{v}}{M_{v}^{0}}\right)^{2} \sum_{v} J_{i v}^{2},
$$

which is represented in the second sum. The $\mathrm{Fe} k_{\mathrm{Fe}}^{(0)}$ and $\mathrm{Pt} k_{\mathrm{Pt}}^{(0)}$ single-ion contributions are found to be $k_{\mathrm{Fe}}^{(6)}=$ $-0.097 \mathrm{meV}$ and $k_{\mathrm{Pt}_{\mathrm{t}}}^{(0)}=1.427 \mathrm{meV}^{17}$

The dipole-dipole coupling is represented in the third sum, while the final term describes the coupling of the magnetic moments to an external magnetic field $\mathbf{B}$. Note that all parameters follow from SDFT calculation are described in detail in Ref. 17 so that the model contains no adjustable parameters.

In our simulation, we use Langevin dynamics, i.e., simulations of the stochastic Landau-Lifshitz-Gilbert equation of motion,

$$
\dot{\mathbf{S}}_{i}=-\frac{\gamma}{\left(1+\lambda^{2}\right) \mu_{\mathrm{s}}}\left[\mathbf{S}_{i} \times \mathbf{H}_{i}+\lambda \mathbf{S}_{i} \times\left(\mathbf{S}_{i} \times \mathbf{H}_{i}\right)\right]
$$

with the internal field $\mathbf{H}_{i}=-\frac{\partial H}{\mathbf{S}_{i}}+\zeta_{\mathrm{i}}(\mathbf{t})$. Thermal fluctuations are included as an additional noise term in the internal fields with $\left\langle\zeta_{i}(t)=0\right\rangle$ and $\left\langle\zeta_{i}^{k}(t) \xi_{i}^{\prime}\left(t^{\prime}\right)\right\rangle=2 \delta_{i j} \delta_{k l} \delta\left(t-t^{\prime}\right) \lambda k_{B} T \mu_{s} / \gamma$, where $i, j$ denotes lattice sites and $k, l$ the Cartesian components. All algorithms that we use are described in detail in Ref. 14.

First of all, our aim is a calculation of parameterized equilibrium functions for $m_{e}(T), \tilde{\chi}_{\|}(T)$, and $\widetilde{X}_{\perp}(T)$. These functions are needed as input for the macrospin model following the LLB equation. Therefore, with the atomistic model, we simulate a cubical system of size $6.15 \times 6.15 \times 9.25 \mathrm{~nm}^{3}$ and calculate the equilibrium properties of the magnetization and the susceptibilities. The magnetization (Fig. 1) clearly shows finite-size effects, which are visible as rounding of the mag- 


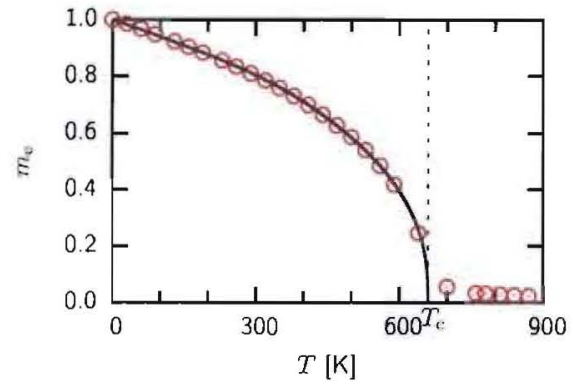

FIG. 1. (Color online) Spontancous equilibrium magnetization vs temperature for the atomistic FePt model. The solid line is a fit to the data extrapolating to $T_{C}$ as for an infinite system.

netization close to the phase transition. However, since the LLB equation implies true critical behavior, we fit our data with a function that extrapolates down to a critical temperature of about $660 \mathrm{~K}$ (solid line), thereby parameterizing a model of infinite system size. This is important since the LLB equation demands a clear definition of the critical temperature. The so parameterized function can then be used as a $m_{e}(T)$ function for the simulations of the LLB equation.

The susceptibilities shown in Fig. 2 are calculated from the fluctuations of the magnetization ${ }^{30}$ given by

$$
\tilde{\chi}_{l}=\frac{\mu_{s} N}{k_{B} T}\left(\left\langle S_{l}^{2}\right\rangle-\left\langle S_{l}\right\rangle^{2}\right),
$$

where $N$ is the number of spins. They clearly exhibit stronger fluctuations than the magnetization and in addition, $\tilde{\chi}_{\mid}$shows once again finite-size effects. As before, we fit our data with functions that extrapolate to the critical behavior of an infinite system, i.e., a divergence of $\tilde{\chi}_{\|}$. Well above $r_{C}$, the two susceptibilities collapse.

The calculation of the thermodynamic exchange stiffness $A(T)$ for the LLB equation is less straightforward. In the following, we use a result derived from the temperature dependent free energy of a domain wall and its corresponding width. The free energy $\Delta F$ of a domain wall is gained from numerical calculations of the internal domain wall energy $\Delta E$, which is the energy difference between a system with and without a domain wall, by using the relation

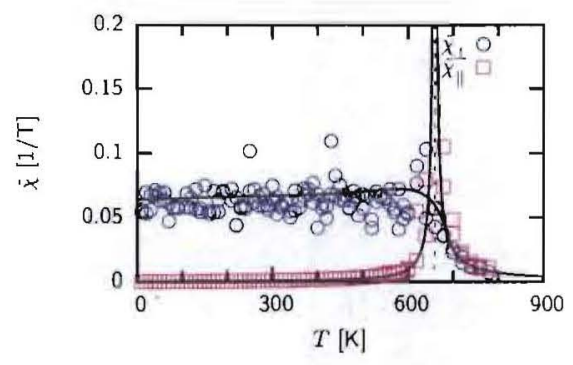

FIG. 2. (Color online) Equilibrium parallel and transverse susceptibility vs temperature for our atomistic FePt model. The solid lines are fits extrapolating the critical behavior.

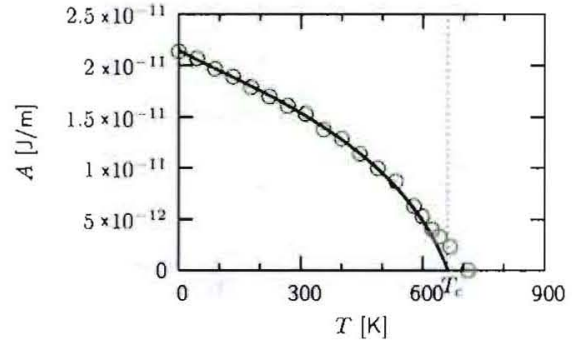

FIG. 3. (Color online) Exchange stiffness vs temperature for the atomistic FePt model. The solid line is a fit extrapolating the critical behavior.

$$
\Delta F(\beta)=\frac{1}{\beta} \int_{0}^{\beta} \Delta E\left(\beta^{\prime}\right) d \beta^{\prime},
$$

with $\beta=1 / k_{B} T$. It was found that domain wall profiles are well described by the usual hyperbolic functions, ${ }^{21}$ so that we were able to fit the domain wall width $\delta$. Assuming that the well-known equations for the domain wall width

$$
\delta(T)=\pi \sqrt{\frac{A(T)}{K(T)}},
$$

and the free energy

$$
\Delta F(T)=4 \sqrt{A(T) K(T)},
$$

temperature dependence holds even at finite temperature, we estimate the micromagnetic exchange stiffness $A(T)$ as well as the anisotropy energy constant $K(T)$. For a detailed description of the calculation, see Refs. 19 and 24 . The corresponding results for $A(T)$ are shown in Fig. 3. Once again, finite size effects can be observed and the fitted line extrapolates down to zero at the Curie temperature.

With $m_{e}(T), A(T), \tilde{\chi}(T)$, and $\tilde{\chi}_{\perp}(T)$, we have all the functions that are necessary as input for the LLB equation. By using these functions, we circumvent further mean-field approximations in the LLB equation and are able to use the microscopic information from the spin Hamiltonian, including the special form of the anisotropy and the long-range exchange interaction of our FePt model. Since the form of the Hamiltonian and all its parameters are derived from SDFT calculations, this approach builds a bridge between electronic degrees of freedom, atomistic spin models, and a macrospin model. In the next section, we will test the macrospin approach versus the original atomistic spin model.

\section{COMPARING ATOMISTIC AND MACROSPIN MODELS}

In the following, the goal is to compare a full atomistic simulation of an FePt nanoparticle by using the Hamiltonian [Eq. (8)] and the numerical methods [Eq. (12)] described above with a much less computation time demanding simulation of a single macrospin by using the LLB equation [Eq. (1)].

We begin the comparison to a longitudinal relaxation shown in Fig. 4. Here, our simulations start with a fully 


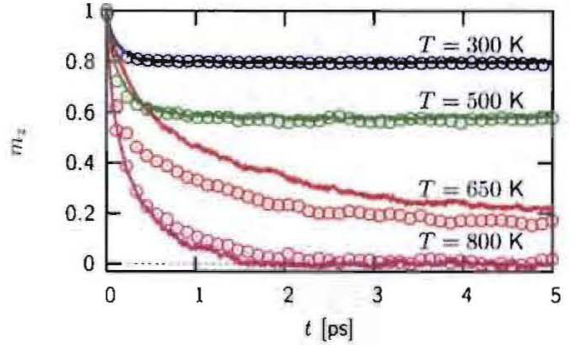

FIG. 4. (Color online) Relaxation of the $z$ component of the magnetization for different temperatures. The data are from an atomistic simulation; the solid lines from a macrospin LLB model $(\lambda$ $=0.1$.

polarized system $\left(S_{z}=1\right)$. Then, the relaxation to its thermal equilibrium value is monitored.

Note that in our simulations, we use a value of $\lambda=0.1$, which is reasonable for a high coercivity medium. It was recently shown ${ }^{31}$ that high values of damping in magnetic media arise from defect-induced magnon-magnon scattering; an effect not included in the current model. Here, we represent the strong dissipation by a large effective $\lambda$.

The agreement between the atomistic model (data points) and the single macrospin (solid lines) model is remarkable, even for $800 \mathrm{~K}$ that is above $T_{C}$. The deviations for $650 \mathrm{~K}$ are due to the fact that here we are close to the Curie temperature. Here, the atomistic simulations show finite size effects leading to shorter (less divergent) relaxation time while for the macrospin following the LLB equation, we used parameterized functions describing the infinite system. Nevertheless, when assuming the same finite volume for the macrospin, even the fluctuations that are due to the finite system size are comparable. Note that this kind of longitudinal relaxation could not at all be described by a macrospin model following the LLG equation of motion, which would keep the length of the magnetization vector constant. Hence, this first test is already far beyond the abilities of conventional micromagnetics.

As a next test, we focus on transverse relaxation. Here, we first equilibrate the system and then we tilt it by an angle of $30^{\circ}$ away from the easy axis. Then we monitor the transverse relaxation shown in Fig. 5. Once again, the agreement between atomistic (data points) and single macrospin (solid lines) model is remarkable. The deviations at the highest

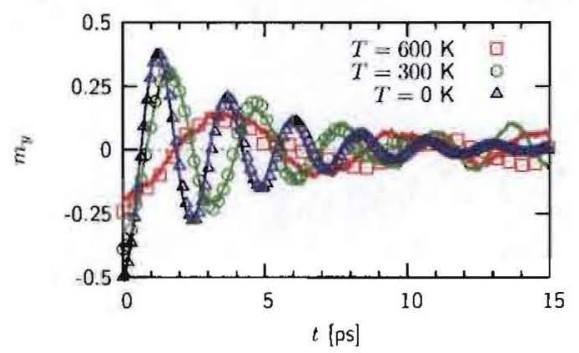

FIG. 5. (Color online) Relaxation of one of the transverse components of the magnetization for different temperatures. The data are from an atomistic simulation; the solid lines from a macrospin LLB model $(\lambda=0.1)$.

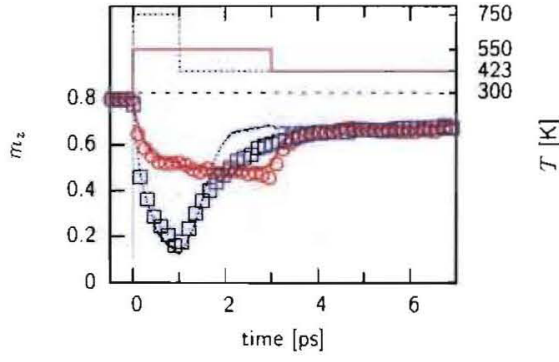

FIG. 6. (Color online) $z$ component of the magnetization vs time for two different pulse heights $(750$ and $550 \mathrm{~K}$ ) with different pulse durations ( 1 and $3 \mathrm{ps}$ ) for atomistic (symbols) and single macrospin simulations (lines). In one case, the pulse height is below $T_{C}$ (circles) and in the other one (squares), well above $T_{C}(\lambda=0.1)$.

temperature shown are due to the fact that thermal fluctuations contribute to a stochastic motion so that the two curves cannot be directly compared on longer time scales.

As mentioned before, the tests we showed before are far beyond the abilities of conventional micromagnetism. In Sec. $\mathrm{V}$, we focus on LLB simulation of fast heating dynamics to show the capability of our approach and to reveal its limits.

\section{FAST HEATING DYNAMICS}

In the following, we compare a full atomistic simulation of fast heating dynamics of an FePt nanoparticle to a simulation of a single macrospin by using the LLB equation. We start our simulation at $300 \mathrm{~K}$ and after a waiting time of some picoseconds, a temperature pulse is applied. Later on, the system is cooled down to $423 \mathrm{~K}$. This rectangular shaped temperature pulse is a simplification of electron temperature profiles as they occur in pump-probe experiments. ${ }^{32}$

Figure 6 shows the response of the magnetization to this step heat pulse for two different peak temperatures and durations, calculated with the atomistic as well as a single macrospin LLB model. The results for the single macrospin model are in good agreement with the atomistic one as long as the heat pulse temperature stays below $T_{C}$ [see the magenta line (LLB) and circles (LLG) in Fig. 6]. In the case wherein the temperature rises above $T_{C}$, the models still show agreement during the demagnetization but deviations occur during recovery [see the blue line (LLB) and squares (LLG) in Fig. 6]. In this temperature range, the atomistic dynamics shows a slower recovery due to multiple nucleation events temporarily leading to a nonuniform magnetization with a much slower reordering dynamics (for details see Ref. 32). This kind of dynamics cannot be described with a single macrospin model.

However, these effects can be taken into account by using a multimacrospin approach. Therefore, we simulate a system of $16 \times 16 \times 24$ macrospins with a cell size $\Delta$ of $3 \mathrm{~nm}$. The exchange between the single grains is taken into account via the exchange stiffness $A(T)$. In the following, we show only results for multimacrospin simulations and not the corresponding atomistic ones, since the considered system size of $48 \times 48 \times 72 \mathrm{~nm}^{3}$ is far beyond the abilities of the full atomistic simulations of an FePt nanoparticle. 


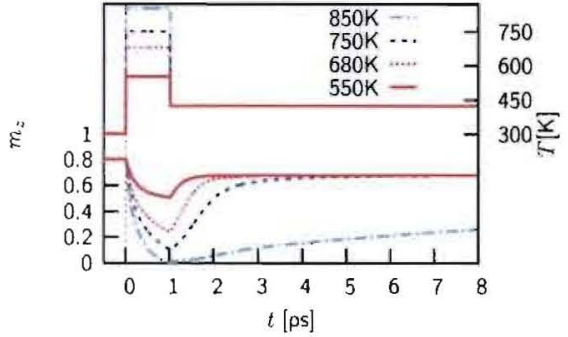

FIG. 7. (Color online) $z$ component of the magnetization vs time for different values of $\lambda$ for a $16 \times 16 \times 24$ macrospin model with $\Delta=3 \mathrm{~nm}$. The pulse width is in all cases $1 \mathrm{ps}$ and the height is varicd from $550 \mathrm{~K}$ up to $850 \mathrm{~K}(\lambda=0.1)$.

Figure 7 shows the time dependence of the magnetization for various values of the peak temperature. As discussed in Ref. 32, the magnetization shows a much slower recovery after full demagnetization, which is essentially due to frustration between multiple volumes of nucleated magnetization caused by the laser pulse. Our multimacrospin simulations clearly show the expected behavior.

\section{SUMMARY AND OUTLOOK}

We introduced a multiscale modeling approach capable of describing thermodynamic equilibrium and nonequilibrium properties of magnetic materials on length scales up to mi- crometers, starting from first principles. In the first step, we modeled bulk FePt in the ordered $\mathrm{Ll}_{0}$ phase by using an effective, classical spin Hamiltonian that was constructed on the basis of first-principles methods. The temperaturedependent micromagnetic parameters, evaluated with these atomistic simulations, were then used as input for many macrospin micromagnetic approach, based on the LandauLifshitz-Bloch equation.

As our tests show, a single macrospin is already capable of reflecting many aspects of an atomistic simulation. In order to see more complicated phenomena resting on nonuniform magnetization states as, e.g., the slow recovery after full demagnetization, it is necessary to consider a multimacrospin approach where the exchange between the grains is taken into account. By using this approach, the numerical simulation of the thermodynamic bchavior of magnetic systems on length scales, which so far were only accessible with conventional micromagnetic approaches, should be possible.

\section{ACKNOWLEDGMENTS}

The authors acknowledge financial support by Seagate Research, Pittsburgh, PA. The use of the White Rose Grid computational resources at the Universities of York and Sheffield is gratefully acknowledged. This work was also partially supported by the common project between Royal Society (United Kingdom) and CSIC (Spain). *un500@york.ac.uk

'E. Bcaurepaire, J.-C. Merle, A. Daunois, and J. Y. Bigot, Phys Rev. Lett. 76, 4250 (1996).

${ }^{2}$ J. Hohlfeld, E. Mathias, R. Knorren, and K. H. Bennemann, Phys. Rev. Lett. 78, 4861 (1997).

${ }^{3}$ A. Scholl, L. Baumgarten, R. Jacquemin, and W. Eberhardt, Phys. Rev. Lett. 79, 5146 (1997).

${ }^{4}$ M. Aeschlimann, M. Bauer, S. Pawlik, W. Weber, R. Burgermeister, D. Oberli, and H. C. Siegmann, Phys. Rev. Lett. 79, 5158 (1997).

${ }^{5}$ B. Koopmans, M. van Kampen, J. T. Kohlhepp, and W. J. M. de Jonge, Phys. Rev. Lett. 85, 844 (2000).

${ }^{6} \mathrm{H}$. Regensburger, R. Vollmer, and J. Kirschner, Phys. Rev. B 61, $14716(2000)$.

${ }^{7}$ G. Zhang, W. Hübner, E. Beaurepaire, and J.-Y. Bigot, in Spin Dynamics in Confined Magnetic Structures I, edited by B. Hillebrands and K. Ounadjela (Springer-Verlag, Berlin, 2002), p. 245.

${ }^{8}$ T. Gerrits, H. A. M. van den Berg, J. Hohlfeld, L. Bär, and T. Rasing, Nature (London) 418, 509 (2002).

${ }^{9}$ B. Koopmans, H. H. J. E. Kicken, M. van Kampen, and W. J. M. de Jonge, J. Magn. Magn. Mater. 286, 271 (2005).

${ }^{10}$ B. Koopmans, J. J. M. Ruigrok, F. Dalla Longa, and W. J. M. de Jonge, Phys. Rev. Lett. 95, 267207 (2005).

${ }^{11}$ A. V. Kimel, A. Kirilyuk, P. A. Usachev, R. V. Pisarev, A. M. Balbashov, and T. Rasing, Nature (London) 435, 655 (2005).

${ }^{12}$ W. Chantrell, M. Wongsam, T. Schrefl, and J. Fidler, in Encyclo- pedia of Materials: Science and Technology, edited by K. H. J. Buschow, R. W. Cahn, M. C. Flemings, B. Ilschner, E. J. Kramer, and S. Mahajan (Elsevier, Amsterdam, 2001).

${ }^{13}$ A. Lyberatos, D. V. Berkov, and R. W. Chantrell, J. Phys.: Condens. Matter 5, 8911 (1993).

${ }^{14} \mathrm{U}$. Nowak, in Handbook of Magnetism and Advanced Magnetic Materials, Micromagnetism, edited by $\mathrm{H}$. Kronmüller and $\mathrm{S}$. Parkin (Wiley, Chichester, 2007), Vol. 2.

${ }^{15}$ O. Chubykalo-Fesenko, U. Nowak, R. W. Chantrell, and D. Garanin, Phys. Rev. B 74, 094436 (2006).

${ }^{16}$ D. A. Garanin, Phys. Rev. B 55, 3050 (1997).

${ }^{17}$ O. N. Mryasov, U. Nowak, K. Guslienko, and R. W. Chantrell, Europhys. Lett. 69, 805 (2005).

${ }^{18}$ U. Nowak, O. N. Mryasov, R. Wieser, K. Guslienko, and R. W. Chantrell, Phys. Rev. B 72, 172410 (2005).

${ }^{19}$ D. Hinzke, U. Nowak, O. N. Mryasov, and R. W. Chantrell, Appl. Phys. Lett. 90, 082507 (2007).

${ }^{20}$ J. Kötzler, D. A. Garanin, M. Hartl, and L. Jahn, Phys. Rev. Lett. 71, 177 (1993).

${ }^{21}$ N. Kazantseva, R. Wieser, and U. Nowak, Phys. Rev. Lett. 94, 037206 (2005).

${ }^{22}$ D. A. Garanin and O. Chubykalo-Fesenko, Phys. Rev. B 70, 212409 (2004).

${ }^{23}$ U. Atxitia, O. Chubykalo-Fesenko, N. Kazantseva, D. Hinzke, U. Nowak, and R. W. Chantrell, Appl. Phys. Lett. 91, 232507 (2007).

${ }^{24}$ D. Hinzke, N. Kazantseva, U. Nowak, O. N. Mryasov, P. Asse- 
lin, and R. W. Chantrell, Phys. Rev. B 77, 094407 (2008).

${ }^{25}$ S. Sun, C. B. Murray, D. Weller, L. Folks, and A. Moser, Science 287, 1989 (2000).

${ }^{26}$ O. N. Mryasov, V. A. Gubanov, and A. I. Liechtenstein, Phys. Rev. B 45, 12330 (1992)

${ }^{27}$ A. B. Shick and O. N. Mryasov, Phys. Rev. B 67, 172407 (2003).

${ }^{28}$ S. Okamoto, N. Kikuchi, O. Kitakami, T. Miyazaki, Y. Shimada, and K. Fukamichi, Phys. Rev. B 66, 024413 (2002).
${ }^{29}$ J.-U. Thiele, K. R. Coffey, M. F. Toney, J. A. Hedstrom, and A. J. Kellock, J. Appl. Phys. 91, 6595 (2002).

${ }^{30}$ D. Hinzke, U. Nowak, and D. A. Garanin, Eur. Phys. J. B 16, 435 (2000).

${ }^{31}$ N. Mo, J. Hohlfeld, M. ul Islam, C. S. Brown, E. Grit, P. Krivosik, W. Tong, A. Rebei, and C. E. Patton, Appl. Phys. Lett. 92, 022506 (2008).

${ }^{32}$ N. Kazantseva, U. Nowak, R. W. Chantrell, J. Hohlfeld, and A. Rebei, Europhys. Lett. 81, 27004 (2008). 